\title{
Is Robotic Pancreatic Surgery A Gamechanger?
}

Jon Cardinal*

Department of Surgery, West Virginia University School of Medicine, USA

*Corresponding author: Jon Cardinal, Department of Surgery, West Virginia University School of Medicine, Tel: 304-293-7095; E-mail: jscardinal@hsc.wvu.edu

Received date: April 13, 2016; Accepted date: April 18, 2016; Published date: April 22, 2016

Copyright: ( 2016 Cardinal J. This is an open-access article distributed under the terms of the Creative Commons Attribution License, which permits unrestricted use, distribution, and reproduction in any medium, provided the original author and source are credited.

\section{Editorial}

Despite initial criticism directed towards the surgical pioneers who performed the first laparoscopic cholecystectomies in the mid-1980s in Europe, minimally invasive surgery (MIS) has taken hold and, in many instances, revolutionized the practice of general surgery. The success of laparoscopic cholecystectomy in reducing post-operative pain, hospital length of stay, and the convalescence period following surgery became so overwhelming that by the mid to late 1990s, laparoscopic cholecystectomy had become standard of care. It was not long after this new era in the surgical management of gallstones had been ushered in that surgeons began applying the minimally invasive skills and principles that they had learned and developed in their experiences with laparoscopic cholecystectomy to other routinely performed surgical procedures including appendectomy, colectomy, splenectomy, adrenalectomy, herniorrhaphy, and gastric procedures (including bariatrics), among others. This shift from open to minimally invasive procedures was driven by both patient as well as surgeon preference.

As indications for minimally invasive approaches to common surgical diseases began to expand, concerns lingered, some of which specifically centered on the oncologic adequacy of minimally invasive approaches in the surgical management of common malignant conditions. In the case of colon cancer, multi-center, prospective randomized trials have shown non-inferiority in terms of oncologic outcomes when comparing laparoscopic to open approaches [1-3]. Similar findings for laparoscopic gastrectomy for gastric cancer were published from Japan [4] and in the case of minimally invasive esophagectomy (MIE), a meta-analysis of 17 case control studies comparing MIE to open esophagectomy found no difference in oncologic outcomes between the two surgical approaches for patients with stage II and stage III disease [5].

With the results of these and other such studies emerging, fears regarding the oncologic outcomes that result from applying minimally invasive approaches to cancer surgery were largely assuaged. Despite this fact, as well as the already described growing interest in extending minimally invasive approaches to common surgical procedures, the application of minimally invasive approaches to the management of both benign and malignant conditions of the pancreas has been comparatively slow to take hold. Pure resectional procedures, such as laparoscopic distal pancreatectomy (LDP) with or without splenectomy, were the first to be attempted and described. Many of the same advantages in outcomes such as decreases in blood loss, length of stay and long term wound complications that had been found when comparing other MIS to open procedures also applied in the case of LDP when compared to open [6]. In regards to laparoscopic pancreaticoduodenectomy (LPD), numerous obstacles have encumbered its widespread adoption, the most significant of which are the ability to control hemorrhage from major vessels as well as to reconstruct the biliary and pancreatic ducts with acceptable morbidity.
Since its first description in 1994, only 9 groups have reported more than 10 laparoscopic pancreaticoduodenectomies with only 400 procedures having been reported in the literature in nearly 2 decades. As a summary of the experience of laparoscopic approaches to pancreatic surgery, a recent query of the National Inpatient Sample database between 2000-2011 is revealing in that of the 36, 195 pancreas resections that were performed over the 12 year period included in the query, only 1994 (919 LPD and 530 LDP), or 4\%, were performed laparoscopically [7]. This failure in dissemination of laparoscopy to the field of pancreatic surgery may speak to inherent limitations posed by the laparoscopic platform.

In contrast, an increasing number of reports are being published on the safety and feasibility of robotics as it applies to pancreatic surgery. Advantages in ergonomics, visualization, precision, and dexterity have all been attributed to robotic surgical platforms and instrumentation when compared to standard laparoscopy. It has been proposed that these advantages may allow for MIS approaches to pancreatic surgery (including complex resections), and the attendant benefits of such approaches, to become more broadly applicable. However, given the fact that experience with and results following robotic pancreatic surgery has only begun to emerge in the first half of this decade [8-11], questions regarding the widespread applicability of robotics to pancreatic surgery remain. These questions surround issues ranging from learning curves, outcomes compared to both open and laparoscopic approaches, training and certification and cost effectiveness, among others.

As one would expect, the learning curve for robotic distal pancreactomy (RDP) has been found to be much less steep than robotic pancreaticoduodenectomy (RPD). In fact, Zeh et al. [11] have described their initial experience and the learning curve associated with each procedure. In the case of the first $100 \mathrm{RDP}$ that they performed, 30 of which were for adenocarcinoma, significant reductions in operative times were observed after the first 20 and 40 cases respectively, and the likelihood of readmission was significantly lower after the first 40 cases. Furthermore, their group found that when comparing their first $30 \mathrm{RDP}$ to 94 consecutive historical controls LDP, there were decreased rates of conversion, increased rates of R0 resection and increased lymph node harvests for both benign and malignant disease in the RDP group [12]. As for the RPD learning curve, they found that in the first 200 consecutive cases that they performed, statistically significant reductions in blood loss and conversion to open procedures were observed following 20 cases, incidence of pancreatic fistula following 40 cases and operative times following 80 cases. Furthermore, they found acceptable 90 day outcomes in the final 120 consecutive RPD in their series including transfusion rates (21.7\%); mortality (3.3\%); fistula rates $(6.9 \%)$; readmission rates $(29.2 \%)$; R0 resection rates $(91.4 \%)$; and median LOS and lymph node harvest (9d and 26 nodes), respectively. Despite these findings, questions remain surrounding the broad applicability of 
such single institution experiences as well as what appears to be the fairly steep learning curve for both RDP and RPD described therein, both of which may limit the ability of more than a few select centers to allow trainees to attain sufficient expertise so as to allow for the widespread growth and dissemination of robotic pancreas surgery.

Finally, conflicting reports exist regarding the cost effectiveness of robotic pancreatic surgery. Clearly, the initial capital investment of the robotic platform puts it at an immediate cost disadvantage; however, potential advantages may exist that could possibly positively impact overall cost. Waters et al. [13] evaluated the cost of RDP, LDP, and ODP, taking into consideration the combined cost of the robotic console/system, operative cost, and hospitalization. RDP costs were lower than LDP and ODP mainly because of the reduction in hospital length of stay for the RDP group (4 days $v s 8$ for LDP and 6 for ODP). To the contrary; however, Kang et al. [14] have shown RDP to be more expensive. Therefore, future analysis of larger cohorts from experienced robotic groups is needed before any conclusions regarding cost effectiveness of robotic pancreas surgery can be drawn.

\section{References}

1. Clinical Outcomes of Surgical Therapy Study Group (2004) A comparison of laparoscopically assisted and open colectomy for colon cancer. N Engl J Med 350: 2050-2059.

2. Fleshman J, Sargent DJ, Green E, Anvari M, Stryker SJ, et al. (2007) Clinical Outcomes of Surgical Therapy Study Group. Laparoscopic colectomy for cancer is not inferior to open surgery based on 5-year data from the COST Study Group trial. Ann Surg 246: 655-662.

3. Jayne DG, Guillou PJ, Thorpe H, Quirke P, Copeland J, et al. (2007) Randomized trial of laparoscopic-assisted resection of colorectal carcinoma: 3 year results of the UK MRC CLASICC Trial Group. J Clin Oncol 25: 3061-3068.
4. Kitano S, Shiraishi N, Uyama I, Sugihara K, Tanigawa N, et al. (2007) A multicenter study on oncologic outcome of laparoscopic gastrectomy for early cancer in Japan. Ann Surg 245: 68-72.

5. Dantoc MM, Cox MR, Eslick GD (2012) Does minimally invasive esophagectomy (MIE) provide for comparable oncologic outcomes to open techniques? A systematic review. J Gastrointest Surg 16: 486-494.

6. Jin T, Altaf K, Xiong JJ, Huang W, Javed MA, et al. (2012) A systematic review and meta-analysis of studies comparing laparoscopic and open distal pancreatectomy. HPB 14: 711-724.

7. Ejaz A, Sachs T, He J, Spolverato G, Hirose K, et al. (2014) A comparison of open and minimally invasive surgery for hepatic and pancreatic resections using the Nationwide Inpatient Sample. Surgery 156: 538-547.

8. Giulianotti PC, Sbrana F, Bianco FM, Elli EF, Shah G, et al. (2010) Robotassisted laparoscopic pancreatic surgery: single-surgeon experience. Surg Endosc 24: 1646-1657.

9. Zureikat AH, Nguyen KT, Bartlett DL, Zeh HJ, Moser AJ (2011) Roboticassisted major pancreatic resection and reconstruction. Arch Surg 146: 256-261.

10. Boone BA, Zenati M, Hogg ME, Steve J, Moser AJ, et al. (2015) Assessment of quality outcomes for robotic pancreaticoduodenectomy: identification of the learning curve. JAMA Surg 150: 416-422.

11. Shakir M, Boone BA, Polanco PM, Zenati MS, et al. (2015) The learning curve for robotic distal pancreatectomy: an analysis of outcomes of the first 100 consecutive cases at a high-volume pancreatic centre. HPB 17: 580-586.

12. Daouadi M, Zureikat AH, Zenati MS, Choudry H, Tsung A, et al. (2013) Robot assisted minimally invasive distal pancreatectomy is superior to the laparoscopic technique. Ann Surg 257: 128-132.

13. Waters JA, Canal DF, Wiebke EA, Dumas RP, Beane JD, et al. (2010) Robotic distal pancreatectomy: cost effective? Surgery 148: 814-823.

14. Kang CM, Kim DH, Lee WJ, Chi HS (2011) Conventional laparoscopic and robot-assisted spleen-preserving pancreatectomy: does da Vinci have clinical advantages? Surg Endosc 25: 2004-2009. 